Journal of

Synchrotron

Radiation

ISSN 0909-0495

\title{
Resonant scattering and diffraction beamline P09 at PETRA III
}

\author{
J. Strempfer, S. Francoual, D. Reuther, D. K. Shukla, A. Skaugen, H. \\ Schulte-Schrepping, T. Kracht and H. Franz
}

J. Synchrotron Rad. (2013). 20, 541-549

Copyright (C) International Union of Crystallography

Author(s) of this paper may load this reprint on their own web site or institutional repository provided that this cover page is retained. Republication of this article or its storage in electronic databases other than as specified above is not permitted without prior permission in writing from the IUCr.

For further information see http://journals.iucr.org/services/authorrights.html

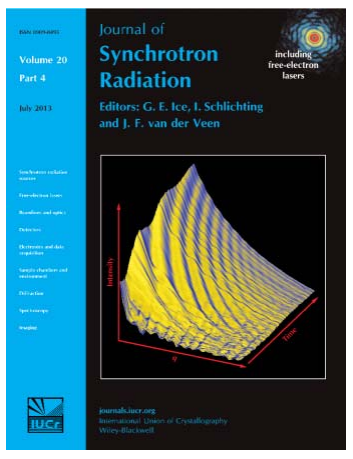

Synchrotron radiation research is rapidly expanding with many new sources of radiation being created globally. Synchrotron radiation plays a leading role in pure science and in emerging technologies. The Journal of Synchrotron Radiation provides comprehensive coverage of the entire field of synchrotron radiation research including instrumentation, theory, computing and scientific applications in areas such as biology, nanoscience and materials science. Rapid publication ensures an up-to-date information resource for scientists and engineers in the field.

Crystallography Journals Online is available from journals.iucr.org 
Journal of

Synchrotron

Radiation

ISSN 0909-0495

Accepted 2 April 2013
Received 5 December 2012

\section{Resonant scattering and diffraction beamline P09 at PETRA III}

\author{
J. Strempfer,* S. Francoual, D. Reuther, D. K. Shukla, A. Skaugen, \\ H. Schulte-Schrepping, T. Kracht and H. Franz
}

Deutsches Elektronen-Synchrotron (DESY), 22603 Hamburg, Germany. E-mail: joerg.strempfer@desy.de

\begin{abstract}
The resonant scattering and diffraction beamline P09 at PETRA III is designed for X-ray experiments requiring small beams, energy tunability, variable polarization and high photon flux. It is highly flexible in terms of beam size and offers full higher harmonic suppression. A state-of-the-art double phaseretarder set-up provides variable linear or circular polarization. A highprecision Psi-diffractometer and a heavy-load diffractometer in horizontal Psigeometry allow the accommodation of a wide variety of sample environments. A $14 \mathrm{~T}$ cryo-magnet is available for scattering experiments in magnetic fields.
\end{abstract}

(C) 2013 International Union of Crystallography Printed in Singapore - all rights reserved

Keywords: resonant scattering; beamline; phase retarder; mirror; diffractometer.

\section{Introduction}

P09 is optimized for resonant and non-resonant elastic scattering and diffraction experiments in the tender to hard X-ray range. Two diffractometers are located in two different experimental hutches: one for standard sample environments such as small cryostats or furnaces and another one for heavy sample environment set-ups. A $14 \mathrm{~T}$ DC magnet is also available. It is equipped with a variable-temperature insert (VTI) and an optional ${ }^{3} \mathrm{He}$ probe. In a third experimental hutch, beamline P09 hosts the hard X-ray photoelectron spectroscopy (HAXPES) station. This paper will focus exclusively on the resonant scattering and diffraction (RSD) stations.

The beamline is dedicated to resonant X-ray diffraction (RXD) experiments, in which the scattering cross section is strongly polarization dependent. However, various scattering and diffraction experiments in need of high photon flux, variable energy, variable polarization and focused beam are also possible. This includes investigation of reflectivities or superlattice reflections as a function of temperature, electric or magnetic fields in bulk samples, thin films or multilayer systems using linear or circular polarization, as well as in situ characterization of sample growth in heavy chambers. With the highly focused X-ray beam, diffraction experiments from small single crystals or single domains within a larger multidomain sample are possible.

The RXD technique is nowadays widely used in various fields (Vettier, 2012) and has been applied extensively to the investigation of electronic order in solids (Beale et al., 2012). By tuning the X-ray energy close to an absorption edge, virtual multipole transitions are induced and the scattering process becomes sensitive to the intermediate states into which the core electrons are excited. From this a strong sensitivity to local environments and asymmetries in the electron distribution arises which can have various origins, such as charge, spin or multipolar order, and can give rise to diffracted intensity at structurally forbidden positions in reciprocal space or an enhancement of weak magnetic reflections.

The anisotropic tensor of susceptibility (ATS) (Dmitrienko, 1983) describes the polarization-dependent nature of the diffracted intensities at forbidden reflections at the absorption edges. ATS scattering was originally applied exclusively to the investigation of purely structural anisotropies. Independent from that, magnetic RXD was discovered (Gibbs et al., 1988), which yields, dependent on the type of transitions, strong resonant enhancement of the weak magnetic intensities of magnetic superlattice reflections. The sensitivity of RXD to magnetic order in the following years was extensively used to investigate magnetic ordering phenomena as a function of temperature, external magnetic field or pressure as a complementary probe to neutron diffraction. The element sensitivity of RXD was used to distinguish between contributions of different elements to the magnetic ordering. ATS scattering was later applied to orbital and charge ordering (Murakami et al., 1998) and since then also to the investigation of higher multipolar order terms.

From the original description of the resonant magnetic cross section (Blume, 1985) until now, theory has advanced considerably and the description of the different resonant diffraction phenomena has been greatly unified by the socalled multipole expansion of the electric and magnetic state of matter (Di Matteo, 2012). This provides a wide field of ordering phenomena to be investigated.

At P09, all requirements for state-of-the-art resonant and non-resonant scattering experiments are met, which include energy tunability, the possibility of vertical as well as hori- 
zontal scattering, manipulation of incident polarization, analysis of the polarization of the diffracted beam, variable beam focus and higher harmonic suppression in the whole energy range. The beamline covers the absorption edges in the energy range from 2.7 to $24 \mathrm{keV}$, which include the $4 d L$-edges (Mo, Ru, Rh,...), the $5 f M$-edges (U), the $4 f L$-edges, the $3 d$ $K$-edges and the $5 d L$-edges. For experiments at low photon energies, P09 is virtually windowless except for a $20 \mu \mathrm{m}$ diamond window which separates the ring from the beamline vacuum.

\section{Storage ring, undulator and front-end}

Beamline P09 is located at the third-generation synchrotron source PETRA III at DESY, which became operational in 2009 (Balewski et al., 2004). The PETRA III storage ring has

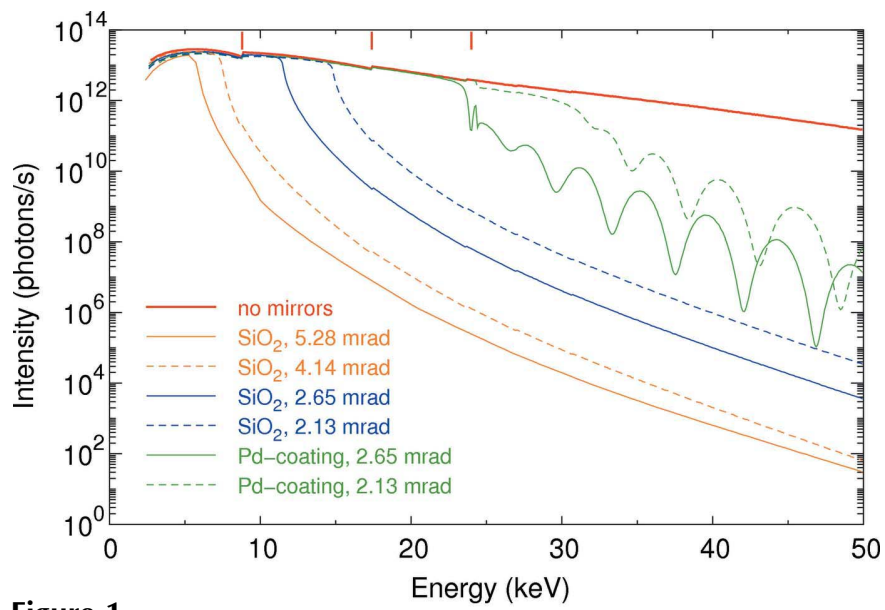

Figure 1

Intensity of direct and mirror-reflected (focused) beam as a function of energy calculated for the U32 spectroscopy undulator, simulated using XTRACE (Seeck, 2006). The solid lines show the curves for a focus in the first experimental hutch (2.65 mrad and 5.28 mrad glancing angles) and the dashed lines show those for a focus in the second experimental hutch (2.13 mrad and $4.14 \mathrm{mrad})$. The corresponding cut-offs are given in Table 1. Vertical lines on top of the spectra mark transitions between undulator harmonics. a circumference of $2.3 \mathrm{~km}$. It accommodates nine straight sections corresponding to nine sectors in the experimental hall covering one-eighth of the ring circumference. In total, 14 beamlines are installed using canted $2 \mathrm{~m}$ undulators with a canting angle of $5 \mathrm{mrad}$ in five sectors, $5 \mathrm{~m}$ undulators in three sectors and a $2 \times 5 \mathrm{~m}$ undulator in the first sector.

PETRA III sets itself apart from other synchrotron sources owing to its low horizontal emittance of $1 \mathrm{~nm}$ rad. The lower emittance directly translates into a reduced horizontal photon source size and beam divergence and into a high brilliance for all beamlines. PETRA III operates at $6.084 \mathrm{GeV}$ ring energy. The ring current is injected in top-up mode with 480 (eventually 960) bunch-filling patterns and $100 \mathrm{~mA}$ ring current in normal operation. Reduced bunch modes with 60 and 40 bunches are used for timing experiments.

P09 is located at sector 6 of the PETRA III experimental hall. It shares the sector with beamline P08, the High-Resolution Diffraction beamline (Seeck et al., 2012). The two beamlines use two separate undulators canted by an angle of $5 \mathrm{mrad}$. The undulators are placed in a high- $\beta$ section of the storage ring, where the calculated r.m.s. photon source sizes and divergences at $8 \mathrm{keV}$ are $140.8 \mu \mathrm{m} \times 5.2 \mu \mathrm{m}(\mathrm{H} \times \mathrm{V})$ and $9.4 \mu \mathrm{rad} \times 6.5 \mu \mathrm{rad}(\mathrm{H} \times \mathrm{V})$, respectively. At P09, a $2 \mathrm{~m}$-long U32 spectroscopy undulator is used with a period length of $\lambda_{\mathrm{U}}=31.4 \mathrm{~mm}$ and a peak field of $B_{0}=0.91 \mathrm{~T}$ (Barthelmess et al., 2008). It generates a continuous energy spectrum via gap scanning with a transition from the first to third harmonic at an energy of $8.8 \mathrm{keV}$ as shown in Fig. 1. The lowest gap size is $9.8 \mathrm{~mm}$, allowing for a minimum energy of $2.7 \mathrm{keV}$ at the first harmonic. Water-cooled power slits in the beamline front-end are used to shape the beam and to reduce the heat load by cutting off the low-energy halo around the central cone.

\section{Beamline optics}

In Fig. 2 we show the different optical elements at P09 and their distances from the source. The optics hutch $\mathrm{P} 09-\mathrm{OH}$ starts at $45.2 \mathrm{~m}$. The first component is the high-heat-load double-crystal fixed-exit monochromator (DCM) (FMB

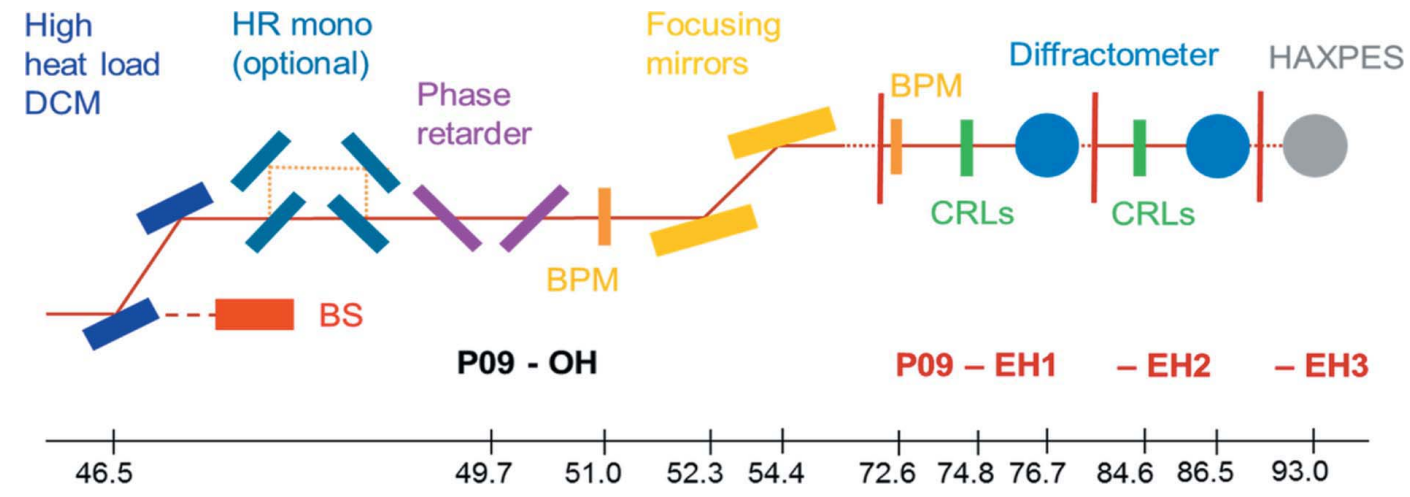

Figure 2

Beam path through the optics of P09 (side view). The scale on the bottom denotes the distance in meters from the source. Left, the high-heat-load double-crystal monochromator is shown together with the beam stop for the white beam (BS). It is followed by the optional high-resolution monochromator foreseen for the HAXPES station. Phase-retarder and focusing mirrors are also located in the optics hutch. On the right, optical components within the first (P09-EH1) and the second experimental hutches (P09-EH2) are shown up to the diffractometers. In the third experimental hutch the position of the HAXPES spectrometer is shown. 
Oxford, Osney Mead, UK) located at $46.5 \mathrm{~m}$. The vertical offset of the monochromatic beam relative to the white beam is $21 \mathrm{~mm}$. A horizontal translation of the whole monochromator tank perpendicular to the beam allows switching between $\mathrm{Si}(111)$ or $\mathrm{Si}(311)$ crystal pairs. Both pairs are cryogenically cooled with liquid $\mathrm{N}_{2}$. Because of the smaller Darwin width of the $\mathrm{Si}(311)$ Bragg reflection, the energy resolution can be improved by almost a factor of five when using $\mathrm{Si}(311)$ instead of $\mathrm{Si}(111)$ at the expense of a factor of five in photon flux. The energy bandwidth is $(\Delta E / E)_{(111)}=1.3 \times 10^{-4}$ and $(\Delta E / E)_{(311)}=0.28 \times 10^{-4}$ or in absolute units $1(0.5) \mathrm{eV}$ and $0.2(0.1) \mathrm{eV}$, respectively, at 8 (4) keV. The lowest energy at which $\mathrm{Si}(311)$ crystal pairs can be used is $5.2 \mathrm{keV}$, corresponding to a maximum monochromator angle of currently 46.7 ${ }^{\circ}$ Quad beam position monitors (QBPMs) (FMB-Oxford) at $51 \mathrm{~m}$ in $\mathrm{P} 09-\mathrm{OH}$ and at $72.6 \mathrm{~m}$ in the first experimental hutch (P09-EH1) equipped with $0.5 \mu \mathrm{m} \mathrm{Ni}$ and Ti foils can be used to stabilize the beam position as well as its direction by adjusting position and pitch of the second monochromator crystal. Downstream of the monochromator at $49.7 \mathrm{~m}$, the double phase-retarder set-up can be used to vary the polarization of the incident monochromatic beam. It is followed by two $1 \mathrm{~m}$-long vertically reflecting mirrors at 52.3 and $54.4 \mathrm{~m}$ used for focusing and higher harmonics rejection. The position of the sample at the center of rotation of the diffractometers is at $76.7 \mathrm{~m}$ in P09-EH1 and at $86.5 \mathrm{~m}$ in P09-EH2. The vacuum at the optical components is maintained at pressures below $10^{-7}$ mbar up to the $20 \mu \mathrm{m}$-thick diamond X-ray window (Diamond Materials, Freiburg) at the entrance to P09-EH1. In P09-EH1, $3 \mathrm{~m}$ in front of the sample position, a third $300 \mathrm{~mm}$ long quartz mirror is available to further suppress higher harmonics in the low-energy regime below $4 \mathrm{keV}$. Additional focusing at fixed energy can be achieved using compound refractive lenses (CRLs) (Lengeler et al., 1999). In the following, the double phase-retarder set-up, the double mirror set-up and the CRL set-up are explained in more detail.

\subsection{Phase retarder}

Magnetic structures are usually determined through investigation of the scattered intensity as a function of the azimuth. However, such measurements imply a rotation of the sample around the scattering vector together with the whole sample environment (i.e. cryostat or magnet) over a large angular range of the azimuth at different scattering vectors which is not always possible. Moreover, different grains of the sample often scatter at different azimuthal angles and thus make such measurements extremely difficult. These difficulties can be overcome using polarization scans, i.e. the generation of incident linearly polarized X-rays at a variable angle $\eta$ of the linear polarization plane around the beam direction and the analysis of the dependence on $\eta$ of the Stokes parameters $P_{1}^{\prime}$ and $P_{2}^{\prime}$ of the diffracted signal from a sample (Blume \& Gibbs, 1988; Detlefs et al., 2012). After its first implementation at beamline ID20 at ESRF (Paolasini et al., 2007), this method has become a routine requirement due to its versatility. Polarization scans allow disentangling between different

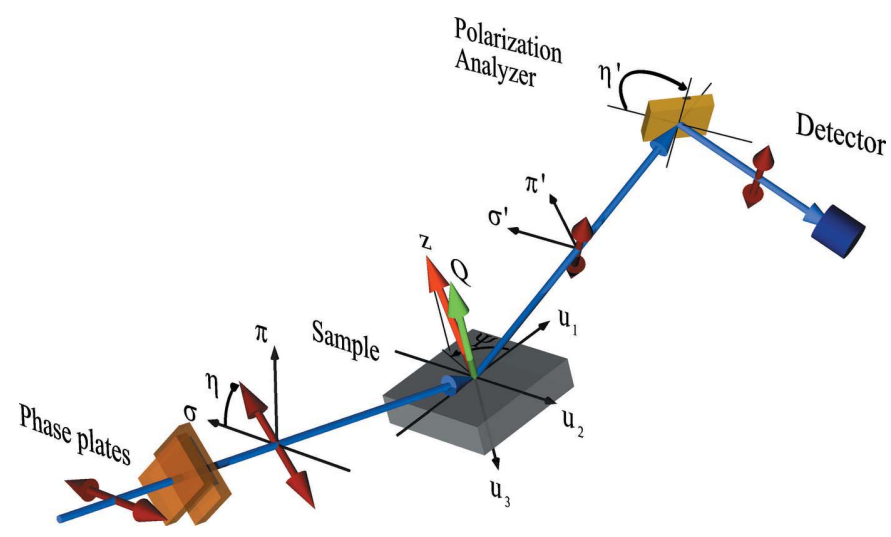

Figure 3

Scattering configuration together with phase plates and polarization analyzer. The phase plates allow a rotation $(\eta)$ of the linear incident polarization in the plane normal to the incident wavevector as well as generation of left and right circular polarization. For the sample the scattering vector $\mathbf{Q}$ and the uniaxial vector $\mathbf{z}$ is shown, which rotates around $\mathbf{Q}$ with the azimuth angle $\Psi$ in the plane normal to the scattering vector. At the sample the reference vectors $\mathbf{u}_{1}, \mathbf{u}_{2}$ and $\mathbf{u}_{3}$ are shown (Blume \& Gibbs, 1988). The scattered signal is analyzed by the polarization analyzer, which can be rotated by the angle $\eta^{\prime}$ around the scattered beam. By rocking the analyzer at several different $\eta^{\prime}$, the polarization properties of the diffracted beam can be determined. Polarization components perpendicular, $\sigma\left(\sigma^{\prime}\right)$, and parallel, $\pi\left(\pi^{\prime}\right)$, to the diffraction plane are shown for the incident (diffracted) X-ray beam.

contributions to the resonant scattering cross section as was first shown by Mazzoli et al. (2007). For magnetic resonant and non-resonant scattering experiments, full polarization scans are used to determine the magnetic moment orientation (Johnson et al., 2008). In addition, circular polarization with the possibility of fast switching between left and right circular polarization is of great importance, since it can be used to determine properties of chiral magnetic structures (Fabrizi et al., 2009). A sketch of a diffraction experiment with phase plates and polarization analyzer is shown in Fig. 3.

Circular and rotated linear polarization can be obtained using quarter-wave plates (QWPs) and half-wave plates (HWPs), respectively. In the hard X-ray regime, QWP and HWP conditions can be obtained by using perfect crystals and slightly detuning them from the ideal Bragg condition (Giles et al., 1994). The actual phase shift depends on the deviation angle from the Bragg position.

The double phase-retarder set-up is mounted in a UHV vacuum tank and consists of two Eulerian cradles $\chi_{\mathrm{pr} 1}$ and $\chi_{\mathrm{pr} 2}$ separated by $340 \mathrm{~mm}$ (Huber Diffraktionstechnik, Rimsting, Germany) (Fig. 4). On each of them two high-resolution rotation stages are mounted opposite to each other acting as $\theta$ and $2 \theta$ circles. The $\theta$ circles are equipped with a $\pm 10 \mathrm{~mm}$ translation making it possible to switch between three phaseretarding plates. A piezo-actuator below the plates allows oscillation with frequencies of up to $40 \mathrm{~Hz}$ for fast switching the helicity of circular polarization. On the $2 \theta$ stages, passivated implanted planar silicon (PIPS) diodes (Canberra) are located which monitor the diffracted intensity. The two Eulerian cradles are mounted on separate $z$ and $x$ stages. Their 


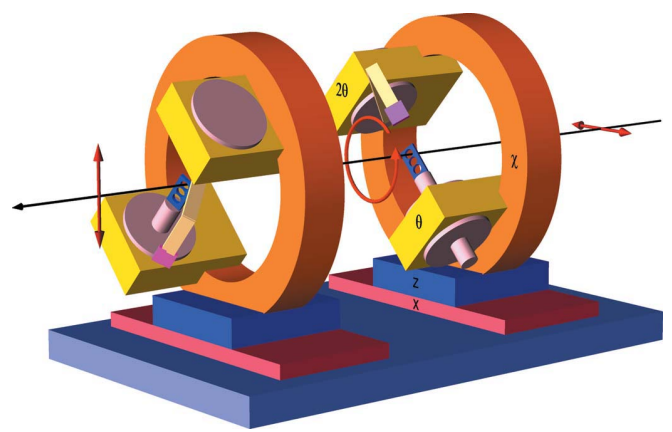

Figure 4

Schematic drawing of the in-vacuum double phase-retarder set-up in the optics hutch. Two individual diffraction stages with opposite $\theta$ and $2 \theta$ circles mounted on the roll $(\chi)$ stages can be seen. Three diamond phase plates are mounted on each of the crystal changers. The inclination of the diffraction plane shown is $\pm 45^{\circ}$ for the two circles. With both phase plates in the QWP condition this results in circular polarization after the first phase plate and $90^{\circ}$ rotated linear polarization after the second phase plate.

axes of rotation are aligned towards each other within a precision of better than $0.1^{\circ}$. The rotation axis of one of them can be aligned precisely along the beam axis using yaw and pitch rotations. As a result, Bragg peaks remain in the diffraction condition within \pm 5 arcsec when $\chi_{\mathrm{pr} 2}$ is rotated between 0 and $-90^{\circ}$ and within $\pm 10 \operatorname{arcsec}$ when $\chi_{\mathrm{pr} 1}$ is rotated between 0 and $+90^{\circ}$.

Currently, two identical sets of synthetic type-Ib diamond phase plates are mounted on the two $\chi_{\mathrm{pr} 1}$ and $\chi_{\mathrm{pr} 2}$ stages: two diamond plates of 100 and $200 \mu \mathrm{m}$ thickness with the [111] direction as surface normal and one diamond plate of $400 \mu \mathrm{m}$ thickness with the [100] direction as surface normal. Each plate is oriented such that the (111) and ( $\overline{1} \overline{1} 1)$ reflections can be set in the diffraction condition, which allows an extension of the usable energy range of each plate owing to the different glancing angles onto the (111) planes. In Fig. 5 the deviation

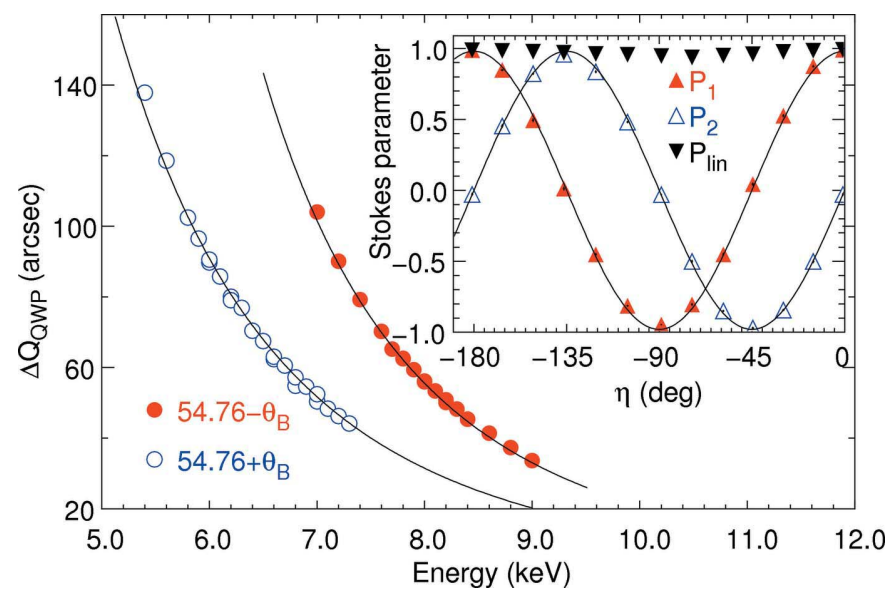

Figure 5

Measured (circles) and calculated (lines) deviation angles for the QWP condition for a $400 \mu \mathrm{m}$ phase-plate for two different incident angles as a function of energy. The insert shows the measured variation of the Stokes parameters with varying angle of linear polarization $\eta$ at $6.5 \mathrm{keV}$ using two QWPs in the $90^{\circ}$ geometry. $P_{\text {lin }}$ gives the degree of linear polarization (inverted filled triangles). angles from the Bragg position necessary to achieve the QWP condition are shown for the $400 \mu \mathrm{m}$ [100] plate for two different glancing angles. Depending on which plate is used and which reflection is set diffracting, the plates are either used in the asymmetric Laue geometry or the symmetric Bragg geometry. The present set of diamond phase plates allows manipulation of the polarization of the incident X-rays in the energy range between 3.5 and $8 \mathrm{keV}$.

Linear polarization scans are carried out using either one single HWP or two QWPs in series and rotating their scattering planes around the X-ray beam. Scagnoli et al. (2009) have shown that using two QWPs in series in a $90^{\circ}$ geometry, i.e. $\chi_{\mathrm{pr} 1}-\chi_{\mathrm{pr} 2}=90^{\circ}$, allows compensation for depolarization effects mostly due to the energy spread, while two QWPs in series in an antiparallel geometry, i.e. $\chi_{\mathrm{pr} 1}-\chi_{\mathrm{pr} 2}$ $=180^{\circ}$, compensate for depolarization effects due to the angular divergence. The angular divergence is very low at P09 and can be neglected. The energy spread, on the other hand, contributes an angular spread of more than $10 \operatorname{arcsec}$ at energies below $9 \mathrm{keV}$, which is non-negligible. The combination of two QWPs in the $90^{\circ}$ geometry results in a $5-10 \%$ higher degree of linear polarization $P_{\text {lin }}$ than (i) using one single HWP of half the total effective thickness (Francoual et al., 2013) and (ii) using two QWPs in the parallel geometry, i.e. $\chi_{\mathrm{pr} 1}=\chi_{\mathrm{pr} 2}$ (Scagnoli et al., 2009; Okitsu et al., 2001; Inami et al., 2013). An example of the direct beam polarization properties for two QWPs in series in the $90^{\circ}$ geometry at the Mn $K$-edge energy is given in the inset of Fig. 5. The degree of linear polarization $P_{\operatorname{lin}}=\left(P_{1}^{2}+P_{2}^{2}\right)^{1 / 2}$ is $96.8(1.7) \%$ on average.

It is foreseen in the near future to change to synthetic typeIIa diamond plates for which QWP deviation angles as low as 20 arcsec can be used (Scagnoli et al., 2009; Okitsu et al., 2001). Right now, the minimum deviation angles for the type-Ib diamond plates used at P09 are 40 arcsec (Francoual et al., 2013). Type-IIa synthetic diamonds produced by the temperature gradient growth method at high temperature and high pressure are better quality than type-Ib diamonds regarding lattice strain and crystal defect structures as well as regarding the amount of impurities and inhomogeneities (Härtwig \& Connell, 2005). A previous study has shown that while synthetic type-IIa diamonds show a typical broadening of the Darwin width of up to 5 arcsec over the whole crystal, synthetic type-Ib diamonds show a width broadening of up to 20 arcsec (Sumiya et al., 1997). A direct benefit will be the larger usable energy range of each crystal and a higher transmission rate at the lowest deviation angles. The type-IIa diamond phase-plates will cover the energy range from 3.2 to $14 \mathrm{keV}$. A set of $10 \mu \mathrm{m}$ thin silicon phase plates will cover the energy range between 2.7 and $3.2 \mathrm{keV}$ (Goulon et al., 1996; Bouchenoire et al., 2012). An additional double phaseretarder set-up allowing operation of two plates in series in the $90^{\circ}$ geometry has been designed to fit at the optical table in P09-EH1 in front of the transfocator and the beam monitor for variable polarization in P09-EH1 and P09-EH2. This device is not in-vacuum and will accommodate the thick type-IIa diamond plates $(t>600 \mu \mathrm{m})$. 
Table 1

Specifications of the mirror system for different glancing angles.

\begin{tabular}{lllllll}
\hline $\begin{array}{l}\text { Focus } \\
\text { position } \\
(\mathrm{m})\end{array}$ & Coating & $\begin{array}{l}r_{1} \\
(\mathrm{~mm})\end{array}$ & $\begin{array}{l}r_{2} \\
(\mathrm{~mm})\end{array}$ & $\begin{array}{l}R \\
(\mathrm{~km})\end{array}$ & $\begin{array}{l}\text { Glancing } \\
\text { angle } \\
(\mathrm{mrad})\end{array}$ & $\begin{array}{l}\text { Cut-off } \\
(\mathrm{keV})\end{array}$ \\
\hline Unfocused & $\mathrm{SiO}_{2}$ & Flat & Flat & Flat & Variable & Variable \\
76.7 & $\mathrm{SiO}_{2}(\mathrm{Pd})$ & 88 & Flat & 11.9 & 2.65 & $11.5(24)$ \\
& $\mathrm{SiO}_{2}$ & Flat & 167 & 6.0 & 5.28 & 5.7 \\
86.5 & $\mathrm{SiO}_{2}(\mathrm{Pd})$ & 88 & Flat & 19.4 & 2.13 & $14.3(31)$ \\
& $\mathrm{SiO}_{2}$ & Flat & 167 & 9.7 & 4.14 & 7.3 \\
90.1 & $\mathrm{SiO}_{2}(\mathrm{Pd})$ & 88 & Flat & 22.8 & 2.00 & $15.1(32)$ \\
& $\mathrm{SiO}_{2}$ & Flat & 167 & 11.1 & 3.90 & 7.9 \\
93.0 & $\mathrm{SiO}_{2}(\mathrm{Pd})$ & 88 & Flat & 23.1 & 1.95 & $15.7(34)$ \\
& $\mathrm{SiO}_{2}$ & Flat & 167 & 12.2 & 3.70 & 8.3 \\
\hline
\end{tabular}

\subsection{Mirrors}

The mirrors in $\mathrm{P} 09-\mathrm{OH}$ are $1 \mathrm{~m}$-long and $130 \mathrm{~mm}$-wide fused silica $\left(\mathrm{SiO}_{2}\right)$ mirrors with average slope errors over $800 \mathrm{~mm}$ length of $<2.5 \mu \mathrm{rad}$ (r.m.s.) sagittal, $<0.8 \mu \mathrm{rad}$ (r.m.s.) meridional and surface roughness $<0.25 \mathrm{~nm}$ (r.m.s.). In order to preserve parallel beam, the first mirror deflects vertically up, the second vertically down. The first mirror (Pilz-Optics, Oberkochen, Germany) has two cylinders with sagittal radii of curvature of $r_{1}=88 \mathrm{~mm}$ and a flat section in between the cylinders. Half of the flat section and one cylinder are coated with $40 \mathrm{~nm}$ palladium. The second mirror (SESO, Aix-enProvence, France) has one cylinder with a sagittal radius of curvature $r_{2}=167 \mathrm{~mm}$ and two flat sections at the sides, where one of them is coated with palladium. In addition, the second mirror is equipped with a bender allowing meridional radii of curvature down to $R=5 \mathrm{~km}$ for vertical focusing. By combining different settings of the two mirrors as shown in Table 1, it is possible to efficiently suppress higher harmonics in the energy range from 6 to $24 \mathrm{keV}$ in P09-EH1 and 7 to $32 \mathrm{keV}$ in $\mathrm{P} 09-\mathrm{EH} 2$, and at the same time to focus the beam into P09-EH1, P09-EH2 and P09-EH3 by varying the glancing angle.

The mirrors are placed in separate UHV chambers. Yaw and pitch rotations and the translations perpendicular to the beam are externally applied to the whole chamber. The pneumatic bender is also external and the bending mechanically transferred into the chamber to the mirror through bellows. The focus size of the mirror-reflected beam at the sample position in P09-EH1 is $150 \times 30 \mu \mathrm{m}$ (FWHM). A vertical knife-edge scan over the focused beam at the sample position in P09-EH1 is shown in Fig. 6(a). The vertical width corresponds to a local meridional slope error of $<0.5 \mu \mathrm{rad}$ (r.m.s.), showing the high quality of some significant mirror regions.

Calculated reflected intensities as a function of energy of the different optical surfaces at different glancing angles are shown in Fig. 1. Because of the fixed distance between the mirrors, the beam height varies with the glancing angle, which means that the components after the mirrors have to be adjusted in height accordingly. After both mirrors, removable CVD diamond screens are located for beam alignment. The intensity of the mirror-reflected beam at the sample position was determined at $8 \mathrm{keV}$ to be $2 \times 10^{13}$ photons s $^{-1}$ using a

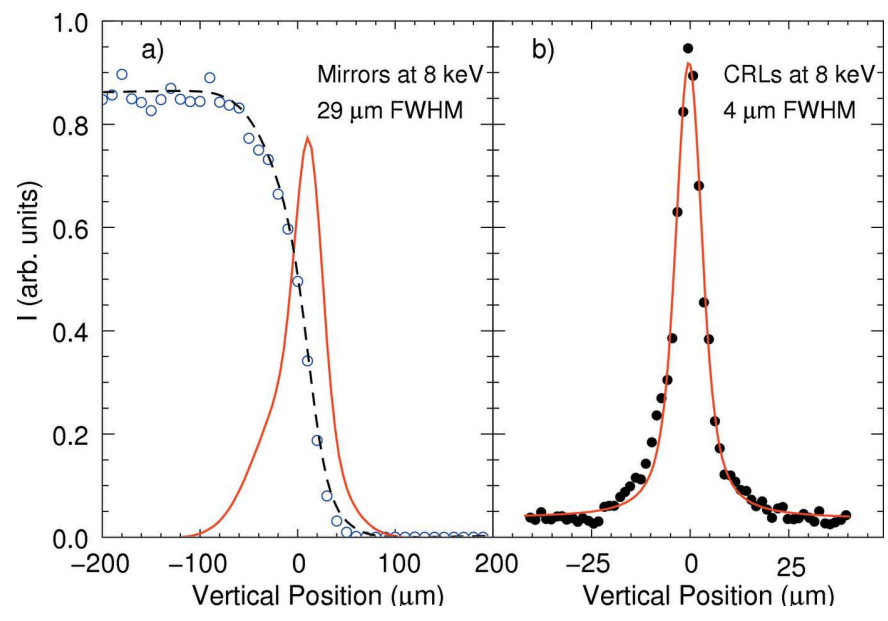

Figure 6

(a) Vertical edge scan over the focused beam at the sample position in P09-EH1 at $8 \mathrm{keV}$ using the mirrors as the focusing device in the $2.65 \mathrm{mrad}$ configuration ( $c f$. Table 1 ). The vertical beam size is $29 \mu \mathrm{m}$. The derivative of the fit to the edge function (dotted line) is shown as a full line. (b) Wire scan (inverted) using a $5.8 \mu \mathrm{m}$ wire over the beam, refocused by compound refractive lenses, positioned $1.9 \mathrm{~m}$ before the sample position in EH1 at $76.7 \mathrm{~m}$, with a virtual source at $93 \mathrm{~m}$ (prefocused by the mirror). The width of $4 \mu \mathrm{m}$ is derived from a fit of a Lorentzian convoluted by the Gaussian representing the wire thickness.

calibrated photodiode. This corresponds well to the calculated value.

The high incident flux is very important when phase plates or CRLs are used in front of the sample, since these reduce the incident flux considerably and flux-hungry experiments such as non-resonant magnetic scattering would not be possible otherwise. However, great care has to be taken for experiments at low temperatures, since beam heating owing to the high photon flux on the sample may affect the sample temperature considerably. Depending on the type of sample (metal, insulator) and the temperature range, a reduction of the incident beam intensity using attenuators in front of the sample might be necessary.

\subsection{Microfocusing with CRLs}

A movable in-vacuum lens changer (transfocator) is positioned in P09-EH1 or in P09-EH2 at $1.9 \pm 0.15 \mathrm{~m}$ in front of the sample position. The transfocator consists of eight pneumatically actuated assemblies of CRLs varying in number and radius in order to allow focusing between 2.7 and $24 \mathrm{keV}$. It is mounted on motorized pitch and yaw rotations and $x, z$ translations to align the optical axis of the CRLs parallel to the beam. It can also be translated by $300 \mathrm{~mm}$ along the beam for fine-tuning of the focal point. The beam is pre-focused to $90.1 \mathrm{~m}$ from the source by the mirrors (glancing angle 2.0 or $3.9 \mathrm{mrad}$ ) in order to adjust the beam size to the $0.9 \mathrm{~mm}$ aperture of the CRLs. The beam is then refocused to the sample position by the CRLs resulting in a demagnification factor of about $8: 1$. Focal sizes of $4 \mu \mathrm{m}$ vertical and $50 \mu \mathrm{m}$ horizontal (FWHM) are achieved, as is shown in Fig. 6(b), which should be compared with calculated values of $4 \mu \mathrm{m}$ vertical and $20 \mu \mathrm{m}$ horizontal. The efficiency for the 
focusing decreases with decreasing energy. While at $8 \mathrm{keV}$ the intensity loss is about a factor of three, the overall intensity loss between unfocused and focused beam is increasing to factors of 20 at $3.5 \mathrm{keV}$ and 25 at $3 \mathrm{keV}$ mainly due to small impurities in the Be lenses.

\section{First experimental hutch (EH1) and six-circle diffractometer}

The first optical element in P09-EH1 is a QBPM with the same specifications as the one in the optics hutch. A UHV-compatible high-precision slit system just after the QBPM is used to define the beam (Dommach et al., 2007). Following the slit system, a movable CVD diamond screen is located. A $20 \mu \mathrm{m}$ diamond window with an opening of $3 \mathrm{~mm}$, which is mounted in a CF flange, separates the ring vacuum from the beamline vacuum. The QPBM, the slit system, the diamond screen and the diamond window are mounted on a granite table equipped with a horizontal and vertical translation to align them in the beam. An in-line electron time-of-flight (eToF) polarization monitor using photoelectrons from a gas target will be positioned on the same table in the near future (Ilchen et al., 2009). This device will allow online monitoring of the incident polarization during polarization manipulation of the incident beam using the phase-retarder set-up.

Downstream from the granite table a $0.9 \mathrm{~m} \times 1.8 \mathrm{~m}$ optical table is positioned on which the vacuum beam path with different components is mounted. A mounting system based on Newport X95 rails and carriers and KF40 flanges allows a very flexible modification of the arrangement of components. All components are designed to operate under vacuum down to $10^{-6}$ mbar. The equipment available on the optical table are the additional silica mirror and the transfocator. A beam monitor is positioned after the transfocator. For the beam monitor a motorized translation is used to insert different thicknesses of glassy carbon foils in the beam (20, 60 and $100 \mu \mathrm{m})$. The foils are rotated by $45^{\circ}$ both towards the beam and the ring plane. Two PIPS diodes with acceptance angles of about $10^{\circ}$ positioned $90^{\circ}$ apart are used to monitor the intensities scattered horizontally and vertically. The monitor is extensively used to determine the QWP and HWP positions of the phase plates. The next component is an absorber box. Using pneumatic actuators, 12 different foils can selectively be inserted into the beam path, attenuating the beam up to at least ten orders of magnitude in the whole energy range of 2.7-30 keV. The last component before the diffractometer is an in-vacuum slit system (JJ-X-ray $\mathrm{A} / \mathrm{S}$, Kgs Lyngby, Denmark) followed by a $100 \mu \mathrm{m}$-thick Be window.

The main instrument in P09-EH1 is a high-precision ' $4 \mathrm{~S}+2 \mathrm{D}$ ' six-circle diffractometer (Huber Diffraktionstechnik) equipped with four circles for the sample $(\chi, \varphi, \theta, \mu)$ and two for the detector $(\delta, \gamma)$ (Fig. 7), all circles being independent. It allows both vertical and horizontal diffraction. The $\chi$-circle has an opening of $15 \mathrm{~cm}$ on the top, allowing for large vertical scattering angles. The $\varphi$ cradle is equipped with a Huber $512.12 \mathrm{M}$ motorized $x y z$-cryostat carrier permitting a full $360^{\circ}$ rotation. The sample stage can sustain a load of up to
Table 2

Parameters of the six-circle diffractometer in P09-EH1.

Spheres of confusion (SOC) are obtained for loaded sample stage and detector arm. The motor name conventions are taken from You (1999).

\begin{tabular}{lllll}
\hline Motor & Range $\left(^{\circ}\right)$ & $\begin{array}{l}\text { Accuracy } \\
(\operatorname{arcsec})\end{array}$ & $\begin{array}{l}\text { SOC } \\
(\mu \mathrm{m})\end{array}$ & Comment \\
\hline$\theta$ & $-10 /+90$ & 0.18 & 30 & Vertical $\theta$ \\
$\delta$ & $-30 /+180$ & 0.36 & 30 & Vertical $2 \theta$ \\
$\mu$ & \pm 30 & 0.18 & 25 & Horizontal $\theta$ \\
$\gamma$ & $-20 /+180 \dagger$ & 0.36 & 60 & Horizontal $2 \theta$ \\
$\chi$ & \pm 90 & 0.36 & 15 & Without cryostat \\
$\varphi$ & \pm 48 & & 30 & With cryostat \\
\hline
\end{tabular}

$\dagger$ Dependent on position of $\mu$.

$12 \mathrm{~kg}$. The reinforced detector arm consists of two motorized linear translation stages separated vertically by an angle of $25^{\circ}$. The point detector set-up with beam collimation and analyzer and the two-dimensional detector are both mounted simultaneously, as shown in Fig. 7. The diffractometer stands on an $x z$-table with a $+60 /-30 \mathrm{~mm}$ vertical and $\pm 20 \mathrm{~mm}$ horizontal translation and a pitch of $\pm 1.5^{\circ}$. The angular ranges along with spheres of confusion for the different circles are shown in Table 2.

The following closed-cycle cryostats are available at P09: a 4 to $450 \mathrm{~K}$ ARS DE-202SG cryocooler ( $8 \mathrm{~K}$ base temperature on sample), a 6 to $800 \mathrm{~K}$ ARS CS-202AG cryocooler and a 1.7 to $300 \mathrm{~K}$ ARS DE-302 cryocooler ( $3 \mathrm{~K}$ base temperature at sample in the absence of exchange gas). A vibration-free $4 \mathrm{~K}$ Cooltran He-flow cryostat is also available. The cryostats limit

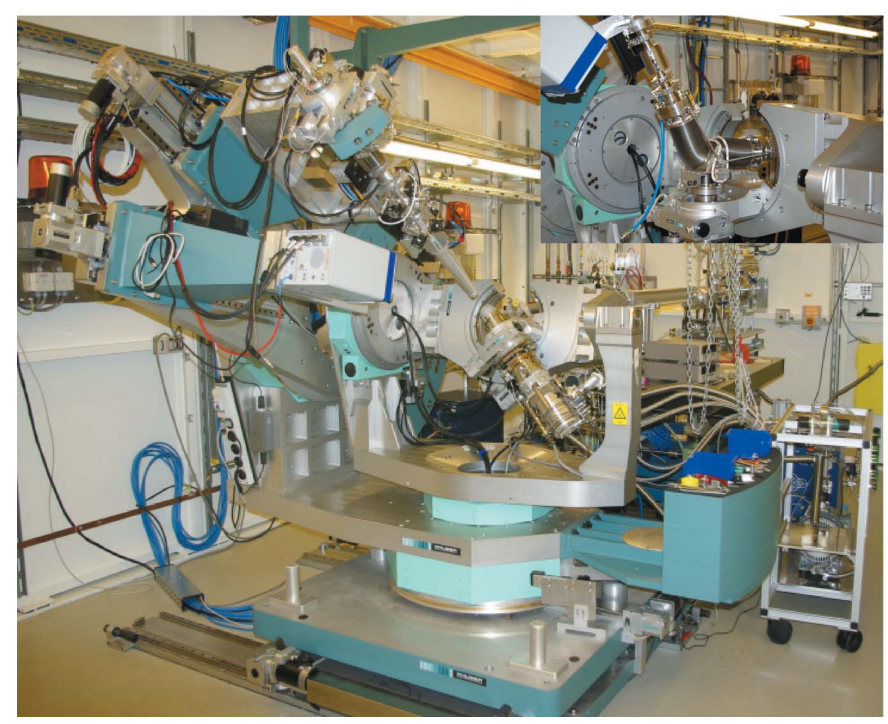

Figure 7

Six-circle diffractometer with open $\chi$-circle. On the double detector arm a point detector together with a polarization analyzer stage and beam collimating slits, as well as a Pilatus $300 \mathrm{k}$ pixel detector, are mounted. Offsetting the scattering angle $\delta$ by $25^{\circ}$ allows switching from measurements with the point detector to measurements with the two-dimensional detector. The $1.7 \mathrm{~K}$ cryocooler is mounted on the $\varphi$ cradle. It is equipped with Be domes. The inset shows the windowless vacuum shroud as replacement for the outer beryllium dome for measurements at energies below $4 \mathrm{keV}$. 
Table 3

Calculated X-ray transmission for different configurations of Be domes (Displex cryostats) and $\mathrm{Be}$ windows (14 $\mathrm{T}$ magnet) as a function of energy.

The first two rows show the transmission without and with the outer dome (D1) in addition to the the radiation shield (D2) for the Displex cryostats. Rows 3 and 4 show the transmission when the dome for exchange gas (D3) is mounted in addition. Rows 5 and 6 show the transmission for the $14 \mathrm{~T}$ magnet without and with ${ }^{3} \mathrm{He}$ insert. Actual values for the low energies might be increased considerably due to impurities.

\begin{tabular}{llllll}
\hline Be $(\mathrm{mm})$ & $8 \mathrm{keV}$ & $6 \mathrm{keV}$ & $4 \mathrm{keV}$ & $2.8 \mathrm{keV}$ & Configuration \\
\hline 0.76 & & 0.71 & 0.32 & $3.3 \times 10^{-2}$ & $\mathrm{D} 2$ \\
1.76 & 0.71 & 0.46 & 0.07 & $3.9 \times 10^{-4}$ & $\mathrm{D} 1, \mathrm{D} 2$ \\
1.52 & & 0.51 & 0.11 & $1.1 \times 10^{-3}$ & $\mathrm{D} 2, \mathrm{D} 3$ \\
2.52 & 0.61 & 0.33 & 0.02 & $1.3 \times 10^{-5}$ & D1, D2, D3 \\
4.0 & 0.45 & 0.18 & $3 \times 10^{-3}$ & $1.7 \times 10^{-8}$ & Magnet \\
6.0 & 0.31 & 0.07 & $1 \times 10^{-4}$ & $2.3 \times 10^{-12}$ & Magnet, ${ }^{3}$ He insert \\
\hline
\end{tabular}

the $\chi$ angular range as shown in Table 2. They are equipped with Be domes: an outer vacuum dome with a wall thickness of $0.5 \mathrm{~mm}$ (D1) and a second dome acting as heat shield with a wall thickness of $0.38 \mathrm{~mm}$ (D2). A third Be dome for exchange gas with a wall thickness of $0.38 \mathrm{~mm}$ (D3) is available for all cryostats. For low-energy experiments the external dome can be replaced by a windowless vacuum shroud to reduce absorption, which becomes relevant for energies below $4 \mathrm{keV}$ (see inset of Fig. 7). At $2.8 \mathrm{keV}$, the intensity gain, when omitting the outer dome, already amounts to a factor of ten for both the incident and diffracted beam as shown in Table 3. The windowless vacuum shroud has angular ranges of $50^{\circ}$ each for the incoming and outgoing beam. The range in $\chi$ is $\pm 5^{\circ}$. An azimuthal rotation is possible through the double O-ring coupling at the cryostat.

On the detector arm a Huber tube cross-slit system 3002.60.M followed by a Huber cross-slit system 3002.70.M are mounted to define the beam path between sample and detector. The connection to the polarization analyzer stage is made using a double O-ring coupling in order to allow an invacuum rotation of the complete analyzer stage around the scattered beam axis ( $\eta^{\prime}$ rotation) and a complete in-vacuum beam path starting at the Kapton window behind the sample up to the detector. The range of $\eta^{\prime}$ is 0 to -150 degrees. The analyzer crystal is mounted with the analyzer Bragg angle of $\theta_{\text {pol }}=45 \pm 5^{\circ}$ towards the diffracted beam. The beam diffracted by the analyzer is recorded by the detector positioned at a scattering angle $2 \theta_{\mathrm{pol}}=90 \pm 8^{\circ}$. The analyzer crystals are attached to the goniometer using kinematic mounts allowing for a fast exchange of the crystals. The height and roll of the analyzer crystals can be adjusted using Attocube piezo stages. A selection of the analyzer crystals available at the beamline together with most relevant absorption edges is shown in Table 4. A Huber two-circle goniometer 415 can be mounted on the $2 \theta$ arm instead of the polarization analyzer stage for experiments requiring higher resolution.

Cyberstar NaI scintillation detectors and APD detectors with ESRF-type amplifiers are available as point detectors at P09 (FMB-Oxford). An energy-dispersive silicon-drift VORTEX EX90 detector (SII NanoTechnology USA,
Table 4

Crystals available for polarization analysis from 2.7 up to $13 \mathrm{keV}$.

Photon energies for $2 \theta=90^{\circ}$ diffraction angle are given for each crystal and reflection, together with some relevant absorption edges close by.

\begin{tabular}{|c|c|c|c|c|}
\hline Crystal & $(h k l)$ & $d(\AA)$ & $\begin{array}{l}E\left(90^{\circ}\right) \\
(\mathrm{keV})\end{array}$ & Absorption edges \\
\hline Graphite & $\left(\begin{array}{lll}0 & 0 & 2\end{array}\right)$ & 3.358 & 2.61 & $\operatorname{Mo}\left(L_{2}\right), \operatorname{Ru}\left(L_{3}\right)$ \\
\hline $\mathrm{TbMnO}_{3}$ & $\left(\begin{array}{lll}0 & 2 & 0\end{array}\right)$ & 2.928 & 2.99 & $\mathrm{Ru}\left(L_{2}\right)$ \\
\hline $\mathrm{Au}$ & $\left(\begin{array}{lll}1 & 1 & 1\end{array}\right)$ & 2.338 & 3.75 & $\mathrm{U}\left(M_{4}, M_{5}\right)$ \\
\hline $\mathrm{Cu}$ & $\left(\begin{array}{lll}1 & 1 & 1\end{array}\right)$ & 2.084 & 4.21 & \\
\hline Graphite & $\left(\begin{array}{lll}0 & 0 & 4\end{array}\right)$ & 1.679 & 5.22 & $\mathrm{~V}(K)$ \\
\hline Mo & $\left(\begin{array}{lll}2 & 0 & 0\end{array}\right)$ & 1.574 & 5.57 & $\mathrm{Ce}\left(L_{3}\right)$ \\
\hline $\mathrm{Al}$ & $\left(\begin{array}{lll}2 & 2 & 0\end{array}\right)$ & 1.432 & 6.12 & $\mathrm{Cr}(K), \operatorname{Pr}\left(L_{3}\right), \operatorname{Nd}\left(L_{3}\right)$ \\
\hline $\mathrm{Cu}$ & $\left(\begin{array}{lll}2 & 2 & 0\end{array}\right)$ & 1.276 & 6.84 & $\begin{array}{l}\operatorname{Mn}(K), \operatorname{Fe}(K), \operatorname{Eu}\left(L_{3}\right), \\
\operatorname{Nd}\left(L_{2}\right), \operatorname{Sm}\left(L_{3}\right)\end{array}$ \\
\hline $\mathrm{Au}$ & $\left(\begin{array}{lll}2 & 2 & 2\end{array}\right)$ & 1.169 & 7.50 & $\operatorname{Tb}\left(L_{3}\right), \operatorname{Sm}\left(L_{2}\right)$ \\
\hline Graphite & $\left(\begin{array}{lll}0 & 0 & 6\end{array}\right)$ & 1.119 & 7.83 & $\operatorname{Dy}\left(L_{3}\right), \operatorname{Gd}\left(L_{2}\right)$ \\
\hline $\mathrm{Cu}$ & $\left(\begin{array}{lll}2 & 2 & 2\end{array}\right)$ & 1.042 & 8.41 & $\operatorname{Dy}\left(L_{2}\right), \operatorname{Tm}\left(L_{3}\right)$ \\
\hline $\mathrm{Pt}$ & $\left(\begin{array}{lll}4 & 0 & 0\end{array}\right)$ & 0.981 & 8.94 & $\mathrm{Cu}(K), \mathrm{Ho}\left(L_{2}\right), \mathrm{Yb}\left(L_{3}\right)$ \\
\hline Graphite & $\left(\begin{array}{lll}0 & 0 & 8\end{array}\right)$ & 0.839 & 10.44 & $\operatorname{Os}\left(L_{3}\right)$ \\
\hline $\mathrm{Au}$ & $\left(\begin{array}{lll}3 & 3 & 3\end{array}\right)$ & 0.779 & 11.25 & $\operatorname{Ir}\left(L_{3}\right)$ \\
\hline Graphite & $\left(\begin{array}{lll}0 & 0 & 10\end{array}\right)$ & 0.671 & 13.05 & $\operatorname{Ir}\left(L_{2}\right), \operatorname{Os}\left(L_{2}\right)$ \\
\hline
\end{tabular}

Northridge, CA, USA) with $25 \mu \mathrm{m}$ Be window is used as a fluorescence detector. A Pilatus 300k two-dimensional pixel detector (Dectris, Baden, Switzerland) is also available at the beamline.

\section{Second experimental hutch (EH2) and heavy-load six-circle diffractometer}

P09-EH2 is equipped with a non-magnetic heavy-load sixcircle diffractometer in horizontal Psi-geometry (Huber Diffraktionstechnik) which is capable of carrying a load of up to $650 \mathrm{~kg}$. There are two independent circles for the detector arm: $\gamma$ (vertical scattering angle) and $\delta$ (horizontal scattering angle), and three for the sample: $\omega$ (horizontal $\theta$ ), $\chi$ and $\varphi$ (Fig. 8). An additional degree of freedom is $\mu$, the limited vertical pitch of $\pm 1^{\circ}$ of the whole diffractometer that moves both sample and detector. The $\chi$ cradle has limited movement of $\pm 7^{\circ}$ for loads of less than $200 \mathrm{~kg}$ while only $\pm 3^{\circ}$ for larger weights. The $x y$ - and $z$-stages allow a $\pm 5 \mathrm{~mm}$ and $\pm 10 \mathrm{~mm}$ horizontal and vertical translation, respectively. The angular ranges along with the spheres of confusion for the different circles are shown in Table 5. The detector arm carries a polarization analyzer stage and two slit systems for beam collimation identical to the set-up in P09-EH1. The slit systems can be removed, making room for the Pilatus 300k area detector. In front of the diffractometer, a multi-purpose nonmagnetic optical table is located (ADC Inc., Lansing, USA), on which the vacuum beam path is mounted. Also here, the transfocator from P09-EH1 can be inserted.

A vertical-field $14 \mathrm{~T}$ split-pair superconducting magnet (Cryogenic Ltd, London, UK) is available in P09-EH2. It is equipped with a variable-temperature insert (VTI) providing temperatures in the range 1.8 to $300 \mathrm{~K}$ in normal operation. A ${ }^{3} \mathrm{He}$ insert is also available and allows access to sample temperatures as low as $300 \mathrm{mK}$. The maximum power deposited on the sample at $8 \mathrm{keV}$ is $26 \mathrm{~mW}$ which has to be 


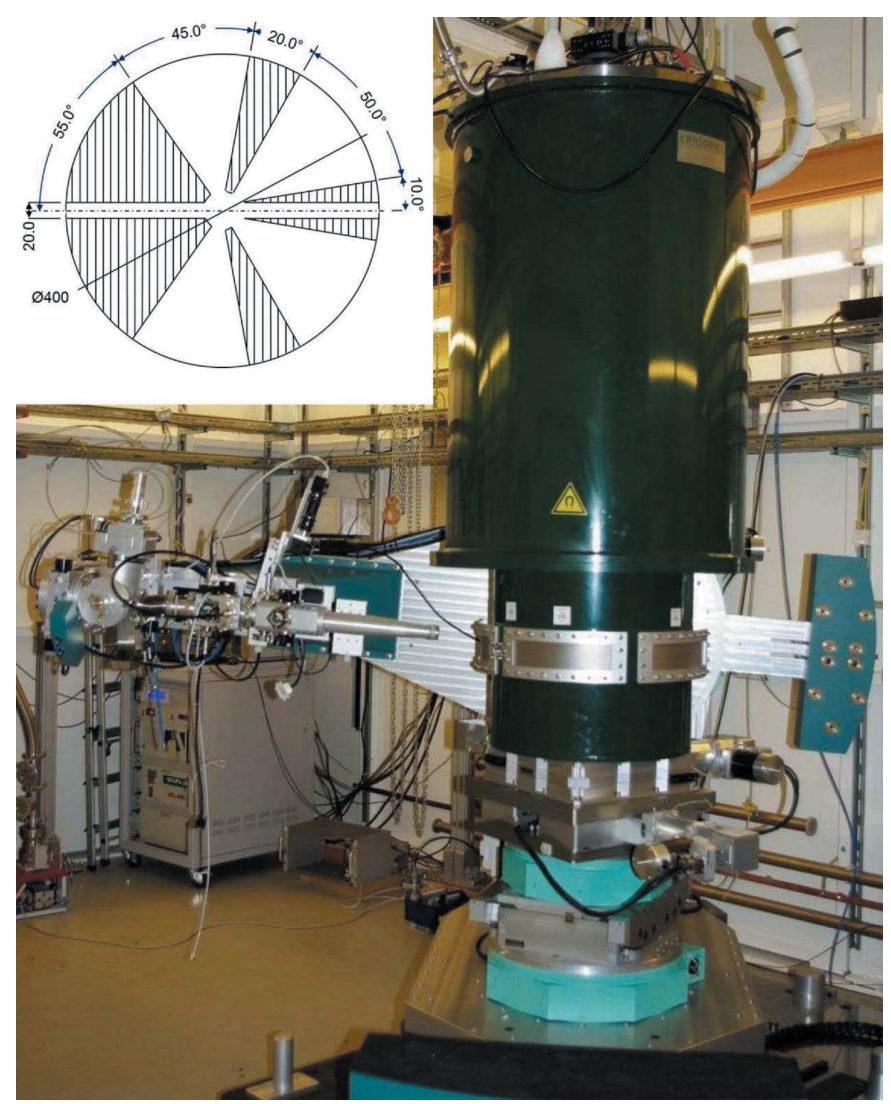

Figure 8

Heavy-load six-circle diffractometer with $14 \mathrm{~T}$ magnet and polarization analyzer. The insert shows the window arrangement of the $14 \mathrm{~T}$ cryomagnet in the horizontal plane.

compared with the cooling power of the ${ }^{3} \mathrm{He}$ stage of $<1 \mathrm{~mW}$. This implies that measurements at these temperatures for a reasonable time are possible only with reduced beam intensities by more than two orders of magnitude.

The X-ray windows around the magnet, at the VTI and the ${ }^{3} \mathrm{He}$ insert, are each made from $1 \mathrm{~mm}$-thick beryllium, in order to keep absorption of the X-ray beam to a minimum. Nevertheless, below $6 \mathrm{keV}$ intensity is already reduced significantly (see Table 3). This limits the usable energy range to energies above $5 \mathrm{keV}$. The vertical opening of the X-ray windows is $\pm 5^{\circ}$ around an aperture of $10 \mathrm{~mm}$. Combining the $\varphi$ and $\theta$ angles and the rotation of the sample probe within the cryostat, the horizontal access has no dark angles, and large horizontal scattering angles of $2 \theta$ up to $170^{\circ}$ can be reached. The horizontal window distribution is shown in the inset of Fig. 8.

\section{Experiment control}

All relevant devices at the beamline are controlled by TANGO, including a diffractometer server for moves in reciprocal space. SPECTRA/ONLINE is the data acquisition and beamline control software used at P09 as a command-line interface between the user and the TANGO devices (Kracht,
Table 5

Parameters of the horizontal Psi-diffractometer in P09-EH2.

Spheres of confusion are obtained for a sample stage loaded with $150 \mathrm{~kg}$ and loaded detector arm. The motor name conventions are taken from You (1999). Compared with the six-circle diffractometer in P09-EH1, vertical and horizontal rotations are swapped.

\begin{tabular}{lllll}
\hline Motor & Range $\left({ }^{\circ}\right)$ & $\begin{array}{l}\text { Accuracy } \\
(\operatorname{arcsec})\end{array}$ & $\begin{array}{l}\text { SOC } \\
(\mu \mathrm{m})\end{array}$ & Comment \\
\hline$\theta$ & \pm 180 & 0.18 & 10 & Horizontal $\theta$ \\
$\delta$ & $-20 /+180$ & 0.36 & 15 & Horizontal $2 \theta$ \\
$\mu$ & \pm 1 & 0.18 & - & Vertical $\theta$ \\
$\gamma$ & $-30 /+180$ & 0.36 & 70 & Vertical $2 \theta$ \\
$\chi$ & \pm 7 & 0.36 & 15 & $<200 \mathrm{~kg}$ \\
$\varphi$ & \pm 3 & & & $650 \mathrm{~kg} \mathrm{load} \mathrm{max}$ \\
\hline
\end{tabular}

2007; Alfaro et al., 2011). A variety of moves and scans are available.

Some TANGO devices special to P09 are mentioned in the following. The multiple motors device allows the definition of slave devices to the energy. For the slave devices a relation between energy and an angular or linear movement is defined, which then moves the slave device whenever the energy is changed. As slave devices, the undulator, the phase retarder and the polarization analyzer can be selected. Like this, energy scans over a peak with arbitrary polarization (e.g. circular) is feasible. The mirror TANGO device allows selection between the several different mirror configurations and moves the two mirrors into position. The phase retarder TANGO devices are used to select between the different phase plates available on each tower and to move the phase plates to $1 / 2,1 / 4$ or $1 / 8$ wave plate condition. The diffractometer device relates the reciprocal space defined by the crystal structure of the sample mounted on the diffractometer to the angular movements of the diffractometer according to the Busing-Levi convention (Busing \& Levi, 1967; You, 1999). The HKL library was developed by F. Picca at SOLEIL. It allows selection between different diffractometer geometries and several different modes of diffraction (bisecting, constant- $\varphi$, constant- $\psi, z$-axis) (Picca, 2011).

\section{Conclusion and outlook}

Beamline P09 has been operational since mid-2010 for external users. Diffraction experiments in both experimental hutches have been performed and show that the equipment is performing according to specification. The investigation of weak non-resonant magnetic reflections in $\mathrm{SmFeAsO}$ shows that very weak signals can be measured reliably (Nandi et al., 2011). Full polarization analysis performed at the Ho $L_{3}$-edge of $\mathrm{HoFe}_{3}\left(\mathrm{BO}_{3}\right)_{4}$ could be used to show the spiral magnetic order along the $c$-axis (Shukla et al., 2012). From azimuthal scans at the Ir $L_{3}$-edge of $\mathrm{Sr}_{2} \mathrm{IrO}_{4}$, the ordering of the $\mathrm{Ir}$ moments in the basal plane could be determined and related to the ordering in $\mathrm{Ba}_{2} \mathrm{IrO}_{4}$ (Boseggia et al., 2013). Application of electric fields and reciprocal-space mapping was used for the investigation of the electric-field-induced superlattice 
reflections from the polar layer at the $\mathrm{LaAlO}_{3} / \mathrm{SrTiO}_{3}$ interface (Rössle et al., 2013).

Above-mentioned representative experiments indicate that P09 combines all the possibilities required for scattering experiments and can be used to perform state-of-the-art experiments in this field. Concerning photon flux, polarization control, polarization analysis, focusing capabilities and high magnetic fields it compares favorably with other experimental stations at third-generation synchrotron sources like APS (4ID-D, 6-ID-B) and SPring-8 (BL22XU) as well as Diamond (I16), ESRF (XMAS) and BESSY (MagS).

The type-IIa diamond phase-plates, together with the installation of the eToF polarization monitor in the near future, will provide means to vary and control polarization in a wide energy range and observe any irregularities during polarization scans, respectively.

The authors would like to acknowledge the staff of the experiment control group FS-EC, M. Hesse and the beamline technology group FS-BT, M. Tischer and the undulator group FS-US, and J. Spengler and the general infrastructure group FS-TI. We also want to acknowledge discussion and advice during the planning of the beamline from O. H. Seeck, J. C. Lang, C. Detlefs, P. Thompson and L. Bouchenoire. Also, we would like to thank K. Pflaum, D. Samberg, A. Gade and R. Döring for engineering and technical support.

\section{References}

Alfaro, M., Flemming, M., Grabitz, J., Kracht, T., Lewendel, B., Nunez, T., van der Reest, P., Rothkirch, A., Schlünzen, F. \& Wintersberger, E. (2011). 13th International Conference on Accelerator and Large Experimental Physics Control Systems (ICALEPCS 2011), 10-14 October 2011, Grenoble, France.

Balewski, K., Brefeld, W., Decking, W., Franz, H., Röhlsberger, R. \& Weckert, E. (2004). PETRA III Technical Design Report. DESY, Hamburg, Germany.

Barthelmess, M., Englisch, U., Pflüger, J., Schöps, A., Skupin, J. \& Tischer, M. (2008). Proceedings of the 11th European Particle Accelerator Conference (EPAC08), Genoa, Italy, p. 2320.

Beale, T., Beutier, G., Bland, S., Bombardi, A., Bouchenoire, L., Bunau, O., Matteo, S. D., Fernandez-Rodriguez, J., HamannBorrero, J., Herrero-Martin, J., Jacques, V., Johnson, R., Juhin, A., Matsumura, T., Mazzoli, C., Mulders, A., Nakao, H., Okamoto, J., Partzsch, S., Princep, A., Scagnoli, V., Strempfer, J., Vecchini, C., Wakabayashi, Y., Walker, H., Wermeille, D. \& Yamasaki, Y. (2012). Eur. Phys. J. Special Topics, 208, 89-98.

Blume, M. (1985). J. Appl. Phys. 57, 3615-3618.

Blume, M. \& Gibbs, D. (1988). Phys. Rev. B, 37, 1779-1789.

Boseggia, S., Springell, R., Walker, H., Rønnow, H., Rüegg, C., Okabe, H., Osobe, M., Perry, R., Collins, S. \& McMorrow, D. (2013). Phys. Rev. Lett. 110, 117207.

Bouchenoire, L., Morris, R. J. H. \& Hase, T. P. A. (2012). Appl. Phys. Lett. 101, 064107.

Busing, W. R. \& Levy, H. A. (1967). Acta Cryst. 22, 457-464.

Detlefs, C., del Rio, M. S. \& Mazzoli, C. (2012). Eur. Phys. J. Special Topics, 208, 359-371.

Di Matteo, S. (2012). J. Phys. D, 45, 163001.

Dmitrienko, V. E. (1983). Acta Cryst. A39, 29-35.

Dommach, M., Franz, H. \& Samberg, D. (2007). HASYLAB Annual Report, pp. 169-170. HASYLAB, Hamburg, Germany.
Fabrizi, F., Walker, H. C., Paolasini, L., de Bergevin, F., Boothroyd, A. T., Prabhakaran, D. \& McMorrow, D. F. (2009). Phys. Rev. Lett. 102, 237205.

Francoual, S., Strempfer, J., Reuther, D., Shukla, D. \& Skaugen, A. (2013). J. Phys. Conf. Ser. 425, 132010.

Gibbs, D., Harshman, D. R., Isaacs, E. D., McWhan, D. B., Mills, D. \& Vettier, C. (1988). Phys. Rev. Lett. 61, 1241-1244.

Giles, C., Malgrange, C., Goulon, J., de Bergevin, F., Vettier, C., Fontaine, A., Dartyge, E. \& Pizzini, S. (1994). Nucl. Instrum. Methods Phys. Res. A, 349, 622-625.

Goulon, J., Malgrange, C., Giles, C., Neumann, C., Rogalev, A., Moguiline, E., De Bergevin, F. \& Vettier, C. (1996). J. Synchrotron Rad. 3, 272-281.

Härtwig, J. \& Connell, S. (2005). Synchrotron Radiat. News, 18, 1519.

Ilchen, M., Deinert, S., Glaser, L., Scholz, F., Seltmann, J., Terka, B., Walter, P., Viefhaus, J., Francoual, S., Reuther, D., Shukla, D. K. \& Strempfer, J. (2009). HASYLAB Annual Report. HASYLAB, Hamburg, Germany.

Inami, T., Michimura, S. \& Matsumura, T. (2013). J. Phys. Conf. Ser. 425, 132011.

Johnson, R. D., Bland, S. R., Mazzoli, C., Beale, T. A. W., Du, C.-H., Detlefs, C., Wilkins, S. B. \& Hatton, P. D. (2008). Phys. Rev. B, 78, 104407.

Kracht, T. (2007). ONLINE, http://hasyweb.desy.de/services/ computing/online/online.html.

Lengeler, B., Schroer, C., Tümmler, J., Benner, B., Richwin, M., Snigirev, A., Snigireva, I. \& Drakopoulos, M. (1999). J. Synchrotron Rad. 6, 1153-1167.

Mazzoli, C., Wilkins, S. B., Matteo, S. D., Detlefs, B., Detlefs, C., Scagnoli, V., Paolasini, L. \& Ghigna, P. (2007). Phys. Rev. B, 76, 195118.

Murakami, Y., Kawada, H., Kawata, H., Tanaka, M., Arima, T., Moritomo, Y. \& Tokura, Y. (1998). Phys. Rev. Lett. 80, 1932.

Nandi, S., Su, Y., Xiao, Y., Price, S., Wang, X. F., Chen, X. H., HerreroMartin, J., Mazzoli, C., Walker, H. C., Paolasini, L., Francoual, S., Shukla, D. K., Strempfer, J., Chatterji, T., Kumar, C. M. N., Mittal, R., Rønnow, H. M., Rüegg, C., McMorrow, D. F. \& Brückel, T. (2011). Phys. Rev. B, 84, 054419.

Okitsu, K., Ueji, Y., Sato, K. \& Amemiya, Y. (2001). J. Synchrotron Rad. 8, 33-37.

Paolasini, L., Detlefs, C., Mazzoli, C., Wilkins, S., Deen, P. P., Bombardi, A., Kernavanois, N., de Bergevin, F., Yakhou, F., Valade, J. P., Breslavetz, I., Fondacaro, A., Pepellin, G. \& Bernard, P. (2007). J. Synchrotron Rad. 14, 301-312.

Picca, F.-E. (2011). HKL, http://people.debian.org/picca/hkl/index. html.

Rössle, M., Kim, K., Dubroka, A., Marsik, P., Wang, C., Jany, R., Richter, C., Mannhart, J., Schneider, C., Frano, A., Wochner, P., Lu, Y., Keimer, B., Shukla, D., Strempfer, J. \& Bernhard, C. (2013). Phys. Rev. Lett. 110, 136805.

Scagnoli, V., Mazzoli, C., Detlefs, C., Bernard, P., Fondacaro, A., Paolasini, L., Fabrizi, F. \& de Bergevin, F. (2009). J. Synchrotron Rad. 16, 778-787.

Seeck, O. H. (2006). HASYLAB Annual Report. HASYLAB, Hamburg, Germany.

Seeck, O. H., Deiter, C., Pflaum, K., Bertam, F., Beerlink, A., Franz, H., Horbach, J., Schulte-Schrepping, H., Murphy, B. M., Greve, M. \& Magnussen, O. (2012). J. Synchrotron Rad. 19, 30-38.

Shukla, D., Francoual, S., Skaugen, A., von Zimmermann, M., Walker, H., Bezmaternykh, L., Gudim, I. A., Temerov, V. L. \& Strempfer, J. (2012). Phys. Rev. B, 86, 224421.

Sumiya, H., Toda, N., Nishibayashi, Y. \& Satoh, S. (1997). J. Cryst. Growth, 178, 485-494.

Vettier, C. (2012). Eur. Phys. J. Special Topics, 208, 3-14.

You, H. (1999). J. Appl. Cryst. 32, 614-623. 